\title{
First detection of a lithium rich carbon star in the Draco dwarf galaxy: Evidence for a young stellar population
}

\author{
I. Domínguez ${ }^{1}$, C. Abia ${ }^{1}$, O. Straniero ${ }^{2}$, S. Cristallo ${ }^{2}$, and Ya. V. Pavlenko ${ }^{3}$ \\ 1 Dpto. Física Teórica y del Cosmos, Universidad de Granada, 18071 Granada, Spain \\ e-mail: inma@ugr.es \\ 2 INAF-Osservatorio Astronomico di Collurania, 64100 Teramo, Italy \\ 3 Main Astronomical Observatory, National Academy of Sciences, Zabolotnoho 27, Kiev-127 03680, Ukranie
}

Received 17 February 2004 / Accepted 29 April 2004

\begin{abstract}
We present a spectroscopic study of D461, a giant star belonging to Draco dwarf spheroidal galaxy. From spectral synthesis in LTE we derive a lithium abundance of $\log \epsilon(\mathrm{Li})=3.5 \pm 0.4$ and a $\mathrm{C} / \mathrm{O}$ ratio between 3 and 5 . This is the first detection of a lithium rich C-star in a dwarf spheroidal galaxy. Basing on stellar models of appropriate chemical composition, we show that a similar $\mathrm{C}$ enrichment is compatible with that expected for a low mass low metallicity thermally pulsing AGB star, undergoing few third dredge up episodes. The position in the $\log g-\log T_{\text {eff }}$ diagram of D461 is also compatible with this theoretical scenario. In particular, the low effective temperature, lower than that expected for a low metallicity giant star, is a consequence of the huge increase of the envelope opacity occurring after the carbon dredge up. The Li enrichment may be explained if a deep circulation would take place during the interpulse period, the so called cool bottom process. In spite of the low resolution of our spectra, we derive a lower limit for the carbon isotopic ratio, namely ${ }^{12} \mathrm{C} /{ }^{13} \mathrm{C}>40$, and a constraint for the $\mathrm{Ba}$ abundance, namely $0.5<[\mathrm{Ba} / \mathrm{Fe}]<2$. The proposed scenario also fits these further constraints. Then, we estimate that the mass of D461 ranges between 1.2 and $2 M_{\odot}$, which corresponds to an age ranging between 1 and $3 \mathrm{Gyr}$. We conclude that this star is more massive and younger than the typical stellar population of Draco.
\end{abstract}

Key words. stars: carbon - stars: abundances - stars: evolution - stars: AGB and post-AGB - galaxies: local group

\section{Introduction}

The origin of the Galactic lithium is still not completely understood. In addition to that produced by the primordial nucleosynthesis, several sources have been invoked to explain the observed abundances in the various components of the Milky Way (see Travaglio et al. 2001, for a recent analysis of the contributions of various possible producers of galactic $\mathrm{Li}$ ). Among these, only for asymptotic giant branch (AGB) stars there are observational evidences of some Li production. $\mathrm{Li}$ is easily destroyed due to the large cross section of its proton capture reaction at the typical temperatures reached inside the stars. As a consequence, giant stars become Li-depleted after the first dredge up. However, it has been early recognized that a Li production may occur in AGB stars via the beryllium convective belt mechanism (Cameron \& Fowler 1971). Two are the conditions to be fulfilled: i) the temperature at the base of the convective envelope must be of the order of $20-30 \times 10^{6} \mathrm{~K}$, so that ${ }^{7} \mathrm{Be}$ can be produced via ${ }^{3} \mathrm{He}+{ }^{4} \mathrm{He}$ reaction, and ii) the mixing must be fast enough to remove the fresh Be from the hot bottom layers, before it decays into Li by electron capture. In such a way, most of the Li will be synthesized in the cool external layers of the star, where the proton capture are defused. Several AGB stars with large abundances of $\mathrm{Li}$ are actually found in the
Galaxy and in the Magellanic Clouds (Catchpole \& Feast 1976; Abia et al. 1993; Smith et al. 1995).

Li enhancement is commonly found in stellar models of massive $\left(M>4 M_{\odot}\right)$ AGB stars as a consequence of the hot bottom burning (HBB; Renzini \& Voli 1981; Sackmann \& Boothroyd 1992; Forestini \& Charbonnel 1997; Lattanzio \& Forestini 1999). However, there are clear indications of an efficient Li production in some low mass AGB stars, where the HBB does not occur. For instance, most of the SLiR ${ }^{1}$ AGB stars found in the Galaxy are carbon stars $(\mathrm{C} / \mathrm{O}>1$, by number, in the envelope, see however García-Lario et al. 1999), while, in the Magellanic Clouds, they are mostly O-rich. Moreover, the luminosities of LiR and SLiR stars in the Clouds are systematically brighter $\left(-6 \leq M_{\text {bol }} \leq-7\right)$ than their galactic counterparts $\left(M_{\mathrm{bol}} \geq-5.5\right)$. Their low luminosity and the fact that $\mathrm{C} / \mathrm{O}>1$ indicate that most of the galactic Li rich stars have low mass progenitors. Finally, the relative abundance of galactic LiR stars with low ${ }^{12} \mathrm{C} /{ }^{13} \mathrm{C}$ ratios $(<15$, mostly C-stars of type J; Abia \& Isern 1997) is substantially larger than that

\footnotetext{
1 We define lithium rich (LiR) and super lithium rich (SLiR) those stars showing $\log \epsilon(\mathrm{Li})>1.0$ and $\log \epsilon(\mathrm{Li})>4.0$, respectively; as usual, $\log \epsilon(\mathrm{X})=\log (\mathrm{X} / \mathrm{H})+12$, where $\mathrm{X} / \mathrm{H}$ is the number of atoms of a given element relative to hydrogen.
} 
measured in the Magellanic Clouds (Hatzidimitriou et al. 2003). In summary, the comprehension of the mechanisms of Li production in different AGB stars as well as its dependence on the parent stellar population deserve further observational and theoretical investigations.

Dwarf galaxies are other stellar systems where AGB stars can be resolved. In the last few years, a growing amount of AGB stars has been discovered in these galaxies: Whitelock et al. (1999) in Sagittarius; Azzopardi et al. (1999) and Demers et al. (2002) in Fornax; Shetrone et al. (2001a) and Margon et al. (2002) in Draco (see also Groenewegen 1999, and references therein). Dwarf galaxies span a wide range in metallicity, so that they could provide new hints about the dependence of the AGB nucleosynthesis ( $\mathrm{Li}$ production included) on the chemical composition of the parent stellar population.

As part of a large survey of AGB stars in the nearby galaxies, we report here, the spectroscopic study of the carbon star D461 in the Draco dwarf spheroidal galaxy, discovered by Armandroff et al. (1995). Let us anticipate our main findings: a) D461 is Li-rich and b) it is more massive and younger than the dominant stellar population of Draco. This is the first report of a star of this type in a dwarf spheroidal. In Sect. 2, we present the observations and the abundance analysis. The evolutionary state of D461 is investigated in Sect. 3, by comparing the available photometric and spectroscopic data to appropriate models of AGB stars. Possible evolutionary scenarios are discussed in the conclusive section.

\section{Observations, lithium identification and analysis}

Spectra of $R \sim 6500$ of several C-stars in Draco were obtained with the ISIS spectrograph attached to the $4.2 \mathrm{~m}$ William Herschel telescope at the Roque de los Muchachos Observatory. The observations were made during July 4-5, 2003. The spectral range covered was $6450-8100 \AA$. D461 was observed during $\sim 4600 \mathrm{~s}$ in several exposures to minimize the impact of the sky background and cosmic rays. The reduction and analysis of the individual spectra was made with the standard techniques using IRAF. The final spectrum has a $S / N \sim 60$ in the region of the $\mathrm{Li} \mathrm{I} \lambda 6708 \AA$ line.

Few notices about Draco $461\left(17^{\mathrm{h}} 19^{\prime} 42.40^{\prime \prime}\right.$; $+57^{\circ} 58^{\prime} 37.8^{\prime \prime}$, Eq. (2000)) can be found in the current literature. Our low resolution spectrum confirm previous identifications of the carbon-rich nature of this star (Armandroff et al. 1995). Very strong $\mathrm{CN}$ bands are clearly seen red-ward to $7850 \AA$ as well as weaker CN bands in the 7000-7200 region. Assuming the visual magnitude given by Shetrone et al. (2001a), $V=17.19$, a distance modulus to Draco of $(m-M)_{o}=19.84 \pm 0.14$ (Bellazzini et al. 2002) and an interstellar extinction $E(B-V)=0.03$ (Mateo 1998), we derive $M_{\mathrm{V}}=-2.74 \pm 0.14$ for D461. Shetrone et al. (2001a) give $(B-V)=1.74$ for this star, which is the reddest color index among the carbon stars so far identified in the Draco galaxy. However, D461 is significantly bluer than the majority of the normal (N-type) carbon stars (therafter, C(N) stars) in the Galaxy. The low metallicity of the Draco stellar population certainly plays a role in determining the photometric properties of its C-stars. Several authors agree for an average metallicity
$\langle[\mathrm{Fe} / \mathrm{H}]\rangle=-2.0$ (Carney \& Seitzer 1986; Bell 1985; Aparicio et al. 2001; Bellazzini et al. 2002), although there is a large dispersion, $-3.0<[\mathrm{Fe} / \mathrm{H}]<-1.5$ (Shetrone et al. 1998). The lack of an IR photometry also limits the knowledge of the effective temperature of D461.

We have evaluated the radial velocity of D461 by crosscorrelating the observed spectra with a theoretical spectrum. This has been done by using the $\lambda 6497 \AA$ Ba II line, the $\lambda 6563 \AA \mathrm{H}_{\alpha}$ line (which is in absorption in D461), and the $\mathrm{CN}$ band heads at $\lambda 7853, \lambda 7876, \lambda 7898$ and $\lambda 7915 \AA$, respectively. The resulting mean radial velocity, $V_{\mathrm{r}}=-301.2 \pm 3.0 \mathrm{~km} \mathrm{~s}^{-1}$, is in good agreement with the value obtained by Armandroff et al. (1995), $V_{\mathrm{r}}=-299.9 \pm 1.5 \mathrm{~km} \mathrm{~s}^{-1}$. After correcting the final observed spectrum for the radial velocity, the strong feature in the lithium region is placed at $\lambda 6707.804 \AA$. Considering the uncertainty in the wavelength calibration $( \pm 10 \mathrm{~m} \AA)$, we unambiguously identify it as the Li I doublet. There are little chances to confuse this feature with the Ce II line at $\lambda 6708.099 \AA$ (Reyners et al. 2002). In this case, in fact, the equivalent width we derive for this line $(\sim 1 \AA)$ would imply an unrealistically large Ce abundance and other very strong features of s-process elements would emerge in the spectrum.

The chemical analysis of D461 is complicated by the lack of a firm determination of its effective temperature. In addition, the low resolution of our spectra does not allow the detection of any unblended metal line to derive the metallicity. Because these difficulties, we computed a grid of model atmospheres for C-rich giants by means of the SAM12 program (see Pavlenko 2003 for details of this code). To account for the frequency and depth distribution of opacity in the atmospheres of late-type stars we used an opacity sampling approach (Sneden et al. 1976). A few line lists from different sources were taken into account: i) line data of diatomic molecules $(\mathrm{CN}$, $\mathrm{C}_{2}, \mathrm{MgH}, \mathrm{H}_{2}, \mathrm{SiH}, \mathrm{CO}, \mathrm{CH}, \mathrm{NH}$ ) were taken from CDROM 18 of Kurucz (1993); ii) atomic lines data were taken from VALD (Kupka et al. 1999); iii) HCN and HNC line lists were computed by Harris et al. (2002); iv) absorption of bands systems of $\mathrm{CaO}\left(\mathrm{C}^{1} \Sigma-\mathrm{X}^{1} \Sigma\right), \mathrm{CS}\left(\mathrm{A}^{1} \Sigma-\mathrm{X}^{1} \Sigma\right)$, $\mathrm{SO}\left(\mathrm{A}^{3} \Pi-\mathrm{X}^{3} \Sigma\right)$, $\mathrm{SiO}\left(\mathrm{E}^{1} \Sigma-\mathrm{X}^{1} \Sigma\right), \mathrm{SiO}\left(\mathrm{A}^{1} \Pi-\mathrm{X}^{1} \Sigma^{+}, \mathrm{NO}\left(\mathrm{C}_{2} \Pi_{r}-\mathrm{X}_{2} \Pi_{\mathrm{r}}\right)\right.$, $\mathrm{NO}\left(\mathrm{B}_{2} \Pi_{r}-\mathrm{X}_{2} \Pi_{\mathrm{r}}\right), \mathrm{NO}\left(\mathrm{A}^{2} \Sigma^{+}-\mathrm{X}_{2} \Pi_{\mathrm{r}}\right), \mathrm{MgO}\left(\mathrm{B}^{1} \Sigma^{+}-\mathrm{X}^{1} \Sigma^{+}\right)$, $\mathrm{AlO}\left(\mathrm{C}^{2} \Pi-\mathrm{X}^{2} \Sigma\right), \mathrm{AlO}\left(\mathrm{B}^{2} \Sigma^{+}-\mathrm{X}^{2} \Sigma^{+}\right)$are taken into account by the JOLA approach (Naersisian et al. 1986).

The following values of the model parameters have been adopted: effective temperature between 3000 and $4000 \mathrm{~K}$ (with a step of $200 \mathrm{~K}), \mathrm{C} / \mathrm{O}=1.05,1.10,3,5$, and 7 , metallicity $[\mathrm{Fe} / \mathrm{H}]=-1.5,-1.7$ and $-2.0, \log g=0$ and microturbulence $\xi=2.5 \mathrm{~km} \mathrm{~s}^{-1}$. Then, we have tried to reproduce the spectrum of D461 in the observed spectral range. As a general rule, the lower the effective temperature, the lower the $\mathrm{C} / \mathrm{O}$ ratio needed to fit the spectrum. Similarly, the higher the metallicity of the model atmosphere, the lower the required $\mathrm{C} / \mathrm{O}$ ratio. After an iteration process, the best reproduction of the observed spectrum of $\mathrm{D} 461$ has been obtained for the following values of the model atmosphere parameters: $T_{\text {eff }}=3600 \pm 200 \mathrm{~K}$, $[\mathrm{Fe} / \mathrm{H}]=-2.0 \pm 0.2$ and $\mathrm{C} / \mathrm{O}$ between 3 and 5 . The estimated value of $T_{\text {eff }}$ is consistent with previous determinations of the effective temperatures for other C-stars in Draco. From infrared photometry, Aaronson \& Mould (1985) derived $T_{\text {eff }}=3800$, 


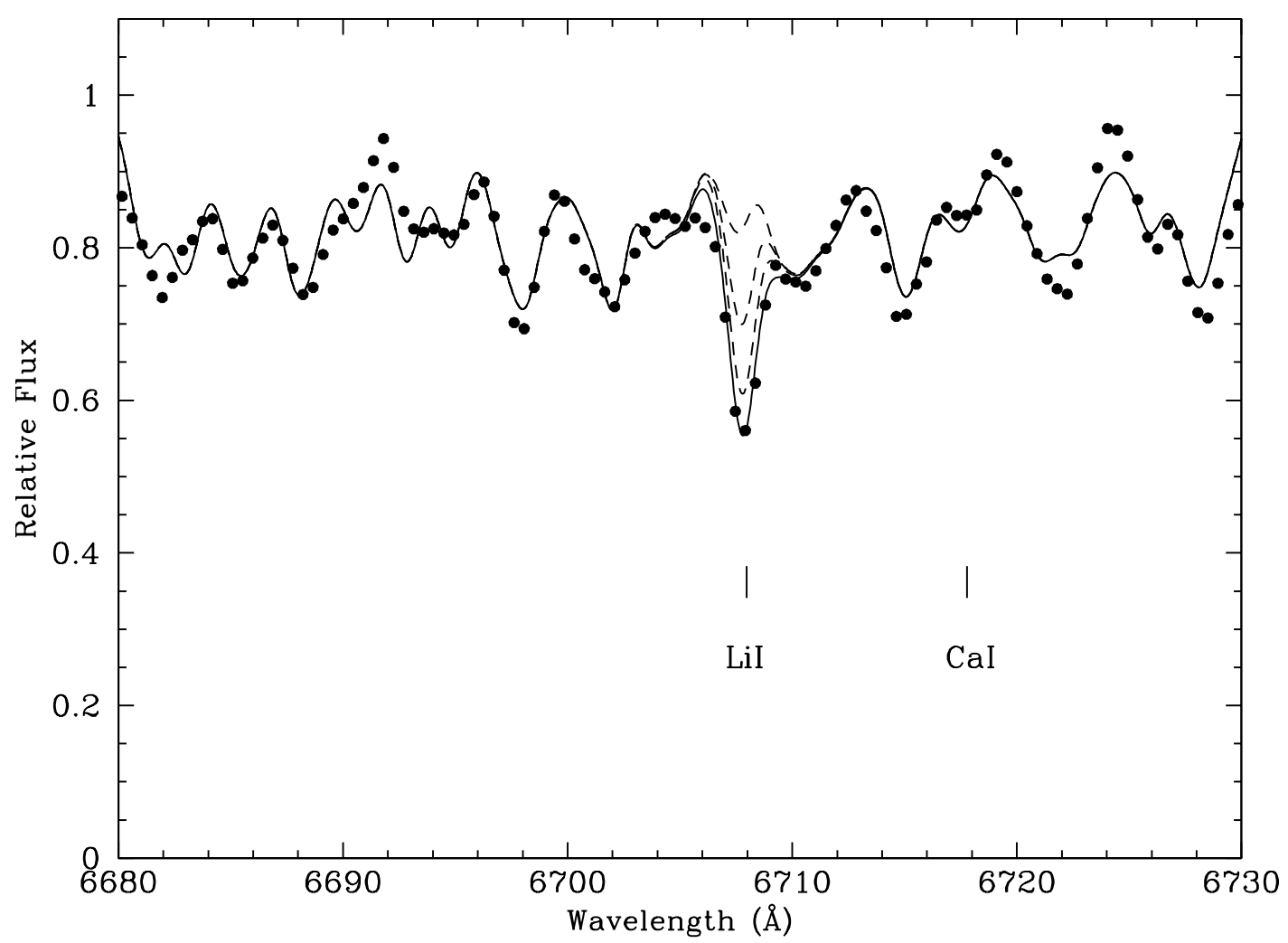

Fig. 1. Synthetic fit to the spectrum of D461 in the lithium region. Solid dots represent the observed spectrum. Lines are synthetic spectra for different $\mathrm{Li}$ abundances: no $\mathrm{Li}, \log \epsilon(\mathrm{Li})=1.5$ and 3.0 (dashed lines) and 3.5 (best fit, continuous line). Theoretical spectra are computed assuming $T_{\text {eff }}=3600 \mathrm{~K},[\mathrm{Fe} / \mathrm{H}]=-2.0$ and $\mathrm{C} / \mathrm{O} \sim 5$. Note that the CaI line at $\sim \lambda 6718 \AA$ is barely seen which it is an evidence of the low metallicity of D461.

3900 and $4100 \mathrm{~K}$ for the stars named $\mathrm{C} 1, \mathrm{C} 2$ and $\mathrm{C} 3$, respectively. These C-stars of Draco have bluer $(B-V)$ index than D461, which is consistent with the lower $T_{\text {eff }}$ value we estimate for it. Note that the clear detection of the $\mathrm{H}_{\alpha}$ line in absorption allow us to put a lower limit to the effective temperature. The cross-correlation of the observed spectra with the theoretical one, ruled out the possibility that this absorption could have a circumstellar origin. Therefore, assuming a photospheric origin for this spectral feature, our tests have revealed that only for $T_{\text {eff }} \geq 3400 \mathrm{~K}$, the $\mathrm{H}_{\alpha}$ line appears in absorption with an appreciable intensity. We recall that $\mathrm{H}_{\alpha}$ in absorption is usually not seen in galactic $\mathrm{C}(\mathrm{N})$ stars. The reason of this is twofold: i) they are cool $\left(T_{\text {eff }} \leq 3000 \mathrm{~K}\right)$, so that the $\mathrm{H}_{\alpha}$ feature is very weak, and ii) the presence of strong blending in the $\lambda 6560 \AA$ region due to intense molecular lines (because of their near solar metallicity). In the case of D461, despite its considerable carbon enhancement, the intensities of molecular lines (mainly $\mathrm{CN}$ and $\mathrm{C}_{2}$ ) are significantly lower in that region of the spectrum. Indeed, there are carbon stars of $\mathrm{CH}$ type in the Galaxy showing $\mathrm{H}_{\alpha}$ in absorption, but they are metal poor and relatively hot, with effective temperatures typically larger than $\sim 4000 \mathrm{~K}$ (Vanture 1992). A preliminary analysis of the $\mathrm{C} 1$ and C3 spectra, both belonging to Draco and observed in the same run as D461, shows also evident $\mathrm{H}_{\alpha}$ features. In particular, a huge emission line is found in the $\mathrm{C} 1$ spectrum, which is a well known symbiotic CH star (Munari 1991), while an absorption line is seen in the C3 spectrum, which is classified as a J-type carbon star. We do not detect the Li line in these two stars.

The Li abundance has been derived by means of the spectral synthesis method. LTE has been assumed. A complete list of atomic and molecular lines $\left(\mathrm{CN}, \mathrm{C}_{2}, \mathrm{CH}\right)$ in a $\sim 50 \AA$ region around the $\mathrm{Li}$ doublet was included in the synthesis (see Abia et al. 2002 for details on this list). Theoretical spectra were convolved with Gaussian functions with a $F W M H \sim 1 \AA$ to simulate the instrumental profile. Figure 1 shows fits to the spectral region of the lithium doublet for different values of the Li abundance. With the atmosphere parameters mentioned above, our best fit to the $\mathrm{Li}$ line is obtained with $\log$ $\epsilon(\mathrm{Li})=3.5$. N-LTE corrections would increase this value by 0.1-0.2 dex (cf. Abia et al. 1999). The main parameter afflicting the $\mathrm{Li}$ abundance derived is $T_{\mathrm{eff}}$. A variation of $\pm 200 \mathrm{~K}$ implies a change in the Li abundance by \pm 0.30 dex. Uncertainties in the $\mathrm{C} / \mathrm{O}$ ratio and the metallicity adopted have much less impact on the estimated $\mathrm{Li}$ abundance. For instance, for the same $T_{\text {eff }}$ and a metallicity $[\mathrm{Fe} / \mathrm{H}]=-1.7$, the estimated abundance would be $\log \epsilon(\mathrm{Li})=3.4$. Finally, a variation of \pm 0.2 dex in the $\log (\mathrm{C} / \mathrm{O})$ have a negligible effect on the estimated Li abundance. Adding quadratically all the errors, including the uncertainty in the continuum location, we estimate a total uncertainty in the $\mathrm{Li}$ abundance of $\pm 0.4 \mathrm{dex}^{2}$.

\footnotetext{
${ }^{2}$ Systematic errors, as those due to possible N-LTE effects, are not
} included. 
In addition to the $\mathrm{Li}$, a search for other chemical peculiarities, which might prove the nature of $\mathrm{D} 461$, has been done. The ${ }^{12} \mathrm{C} /{ }^{13} \mathrm{C}$ ratio is an important test for evolved stars. A low ratio is expected if the envelope material has been exposed to the $\mathrm{CNO}$ burning. $\mathrm{C}(\mathrm{N})$ stars in the Galaxy typically present carbon isotopic ratios in the range 40-60 (Lambert et al. 1986; but see also Ohnaka \& Tsuji 1996; Abia et al. 2002). ${ }^{13} \mathrm{C}$ enhancements can be easily detected by comparing the ratios of ${ }^{13} \mathrm{C}^{12} \mathrm{C} \lambda 4744 \AA$ to ${ }^{12} \mathrm{C}^{12} \mathrm{C} \lambda 4737 \AA$ in the blue or the ratios of ${ }^{12} \mathrm{C}^{12} \mathrm{C} \lambda 6191 \AA$ to ${ }^{13} \mathrm{C}^{12} \mathrm{C} \lambda 6168 \AA{ }^{13} \mathrm{C}^{12} \mathrm{C} \lambda 6102 \AA$ to ${ }^{12} \mathrm{C}^{12} \mathrm{C} \lambda 6122 \AA$ and ${ }^{13} \mathrm{C}^{14} \mathrm{~N} \lambda 6260 \AA$ to ${ }^{12} \mathrm{C}^{14} \mathrm{~N} \lambda 6206 \AA$ at larger wavelengths. Unfortunately, our spectrum did not cover these spectral regions. Then, we tried to derive the carbon isotopic ratio from ${ }^{12,13} \mathrm{CN}$ lines at $\sim \lambda 8000 \AA$ using the same technique as in Abia \& Isern (1996). However, because of the low spectral resolution, only a lower limit has been obtained, namely ${ }^{12} \mathrm{C} /{ }^{13} \mathrm{C}>40$. This limit is not much affected by uncertainties in the stellar metallicity and/or the $\mathrm{C} / \mathrm{O}$ ratio, but it is sensitive to the uncertainty in $T_{\text {eff }}$ : the lower the temperature the higher the carbon isotopic ratio. The quoted lower limit is very conservative, because no $\mathrm{CN}$ feature sensitive to the ${ }^{13} \mathrm{C}$ abundance changes in the $\lambda 8000 \AA$ A region can be fitted with a lower ${ }^{12} \mathrm{C} /{ }^{13} \mathrm{C}$ ratio. Note that there are various ${ }^{13} \mathrm{CN}$ features in the spectrum of D461 that can be adjusted with a considerably larger carbon isotopic ratio (i.e. 100). This latter value would be in better agreement with the theoretical predictions for the ${ }^{12} \mathrm{C} /{ }^{13} \mathrm{C}$ ratio in this star (see next section).

We have also searched for $s$-element enhancements, a clear signature of the AGB nucleosynthesis. The best spectral region for such analysis (4750-4950 $\mathrm{A}$ ) is not accessible in our spectra either and we could only use the Ba II line at $\lambda 6497 \AA$. This line is strong and usually overcrowded with molecular lines in carbon stars of near solar metallicity, so that that it is not useful for abundance analysis in C-stars with such metallicity. However, the low metallicity of D461 helps in reducing the blending, but the incompleteness of the list of molecular lines used in this spectral region reduces our capability of reproducing the Ba blend. An upper limit has been derived: $[\mathrm{Ba} / \mathrm{Fe}]<+2.0$. This limit decreases by increasing the metallicity and the $\mathrm{C} / \mathrm{O}$ ratio or by reducing $T_{\text {eff }}$. For instance, adopting a metallicity $[\mathrm{Fe} / \mathrm{H}]=-1.7$ (keeping constant $T_{\text {eff }}$ and $\mathrm{C} / \mathrm{O}$ ) the upper limit would be $[\mathrm{Ba} / \mathrm{Fe}]<+1.8$. It is also possible to put a lower limit to the $\mathrm{Ba}$ abundance. We have assumed that the spectral feature lacking in the Ba blend was due to a ${ }^{12} \mathrm{CN}$ line. Then, we artificially vary its spectroscopic parameters (excitation energy and $g f$-value) until a fit was obtained to the $\mathrm{Ba}$ blend. The resulting lower limit was $[\mathrm{Ba} / \mathrm{Fe}]>+0.5$ dex.

To summarize, although the chemical characteristics of D461 have to be confirmed by a more detailed analysis based on higher resolution spectra, we can say that this star is certainly Li-rich, with a large carbon enhancement and with a weak evidence of s-process enrichment.

\section{The nature of Draco 461}

Our spectral analysis of D461 reveals several chemical peculiarities worth to be discussed and understood. As far as we know, the estimated $\mathrm{C} / \mathrm{O}$ ratio is the largest ever found for a carbon star. Our tests show that for any reasonable choice of $[\mathrm{Fe} / \mathrm{H}]$ and $T_{\text {eff }}$, the $\mathrm{C} / \mathrm{O}$ ratio in $\mathrm{D} 461$ must be larger than 3 . This value is certainly larger than those typically found in Galactic C-stars, which typically show C/O only slightly above 1 (Lambert et al. 1986; Abia et al. 2002). Recently, Matsuura et al. (2002), by analyzing the intensities of $\mathrm{C}_{2} \mathrm{H}_{2}$ and $\mathrm{HCN}$ bands, found somewhat larger $\mathrm{C} / \mathrm{O}$ ratios $(>1.2)$ in some C-stars of the LMC.

The $\mathrm{C}$ enhancement may be due to the third dredge up (TDU) occurring in thermally pulsing AGB stars. As it is well known, the TDU brings to the surface the ashes of the He burning (essentially carbon) and the products of the neutron capture nucleosynthesis (s-process). The detection of $T \mathrm{c}$, whose s-component has lifetime $\sim 2 \times 10^{5} \mathrm{yr}$, in galactic $\mathrm{C}(\mathrm{N})$ stars proves that they are actually undergoing the third dredge up. Nevertheless, some C-stars could not be AGB stars experiencing the TDU. This may happen in interacting binary systems, when the atmosphere of the secondary (less evolved) star is polluted with the matter lost by an AGB companion (Vanture 1992; Jorissen 1999). The accretion of the less massive component may occur as a consequence of the Roche lobe overflow of the primary (in close systems) or by wind (in loose systems). C-stars of this type are called extrinsic, to be distinguished from the intrinsic C-stars that are AGB stars undergoing the TDU. The depth of the TDU depends on the envelope mass (Iben \& Renzini 1983). Current theoretical models show that there exists a minimum initial mass for the occurrence of the TDU, which varies between 1.3 and $1.5 M_{\odot}$, depending on the metallicity and on the pre-AGB mass loss rate (see e.g., Straniero et al. 2003). This is confirmed by the observational evidence that intrinsic $\mathrm{C}$-stars are not found in very old stellar systems, where stars with mass exceeding this limit are already evolved beyond the AGB. The galactic halo is an example of these old stellar populations, whose carbon rich stars $(\mathrm{CH}$ and dwarf-C) are expected to be extrinsic (Wallerstein \& Knapp 1998). At the metallicity of Draco, even considering a particularly moderate RGB mass loss, the minimum mass for the occurrence of the TDU cannot be lower than $1.2 M_{\odot}$, which corresponds to an AGB age $<4$ Gyr. As in the case of the halo, however, the dominant stellar population in Draco is very old, namely $\sim 10 \mathrm{Gyr}$ (Grillmair et al. 1998). Nevertheless, the abundance pattern of the Draco stellar population differs from that of halo stars with similar metallicity, the most striking peculiarity being the nearly solar, in some cases only slightly enhanced $[\alpha / \mathrm{Fe}]$ ratios (Shetrone et al. 1998, 2001a,b). In addition, the presence of anomalous Cepheids in Draco (Baade \& Swope 1961; Gallart et al. 1999) demonstrates the existence of a more massive (1-2 $M_{\odot}$ ) and younger stellar population. It appears, therefore, that the star formation in Draco would have been extended up to a few Gyrs ago (Aparicio et al. 2001; Ikuta \& Arimoto 2002). Thus, in spite of the large age of its dominant stellar population, it is possible that some C-stars of Draco could be intrinsic AGB stars with masses of the order of $1.5 M_{\odot}$.

By comparing the available photometric and spectroscopic data to extant stellar models of AGB stars, we have looked into the hypothesis of the intrinsic nature of the $\mathrm{C}$ enhancement of 


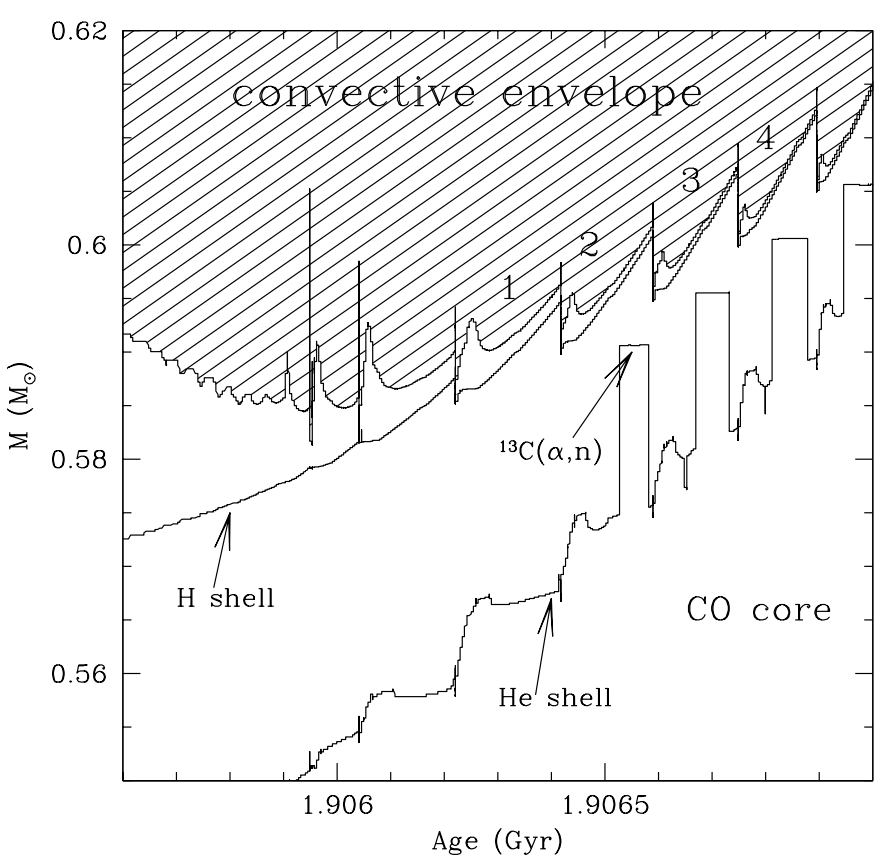

Fig. 2. Evolution of the locations (in mass coordinates) of the maximum energy production in the $\mathrm{H}$ and $\mathrm{He}$ burning shells (solid lines), for a thermally pulsing AGB stellar model with $M=1.5 M_{\odot}$ and $Z=0.0003$. The hatched area shows the lower portion of the convective envelope. Interpulse periods, after the first TDU episode, are numbered as in Table 1. Note that during the interpulse, when the $3 \alpha$ is off, the maximum nuclear energy production moves to the location of the ${ }^{13} \mathrm{C}$ pocket, where the ${ }^{13} \mathrm{C}(\alpha, n){ }^{16} \mathrm{O}$ reaction and the related s-process nucleosynthesis take place.

D461. Then, an evolutionary sequence of models for a star with $M=1.5 M_{\odot}, Z=3 \times 10^{-4}$ and $Y=0.24$ have been computed using the FRANEC code (Chieffi et al. 1998). The calculation was started from the pre main sequence up to the AGB phase. A Reimer's mass loss rate $(\eta=0.4)$ has been adopted since the RGB phase. A time-dependent mixing in the convective zones have been obtained following the algorithm described by Chieffi et al. (2001). At the convective boundaries, an exponential decay of the convective velocity is assumed, which allows the formation of a ${ }^{13} \mathrm{C}$ pocket at the epoch of the TDU (see Cristallo et al. 2004 for further details). Few thermal pulses have been followed in details. In particular, in order to evaluate the nucleosynthesis taking place in the $\mathrm{H}$ and $\mathrm{He}$ burnings shells (s-process included), a full network of 450 isotopes (about 700 reactions) has been coupled to the stellar structure equations. Figure 2 shows the evolution of the locations (in mass coordinates) of the $\mathrm{H}$ and $\mathrm{He}$ burning shells during the TP-AGB phase. The position of the bottom of the convective envelope is also reported. The dredge up occurs when the inflation powered by a thermal pulse (TP) cools the base of the envelope and the $\mathrm{H}$ burning dies down. Starting from the second TP, the bottom of the convective envelope regularly penetrates into the H-exhausted core, down to the region previously enriched
Table 1. Theoretical predictions for the surface composition of D461.

\begin{tabular}{|c|c|c|c|c|c|c|c|}
\hline & $\mathrm{D} 461^{a}$ & Initial & RGB & $1 \mathrm{st}^{b}$ & 2nd & $3 \mathrm{rd}$ & 4th \\
\hline$\Delta M_{\mathrm{up}}^{c}$ & & & & $3 \times 10^{-4}$ & $3 \times 10^{-3}$ & $4 \times 10^{-3}$ & $5 \times 10^{-3}$ \\
\hline $\log \epsilon(\mathrm{Li})$ & $3.5 \pm 0.4$ & & & & & & \\
\hline$\overline{s t d^{d}}$ & & 2.3 & -0.73 & -0.54 & 0.01 & 0.34 & 0.55 \\
\hline 20 & & & & 2.17 & 2.24 & 2.06 & 1.95 \\
\hline 25 & & & & 3.29 & 3.39 & & \\
\hline 30 & & & & 3.62 & 3.76 & & \\
\hline 40 & & & & 3.92 & 3.76 & 3.68 & \\
\hline${ }^{12} \mathrm{C} /{ }^{16} \mathrm{O}$ & $3-5$ & & & & & & \\
\hline$\overline{s t d}$ & & 0.50 & 0.32 & 2.10 & 10.44 & 18.06 & 23.13 \\
\hline 20 & & & & 2.10 & 10.44 & 18.06 & 23.13 \\
\hline 25 & & & & 2.10 & 10.44 & & \\
\hline 30 & & & & 2.09 & 10.44 & & \\
\hline 40 & & & & 1.79 & 9.62 & 15.79 & \\
\hline${ }^{12} \mathrm{C} /{ }^{13} \mathrm{C}$ & $>40$ & & & & & & \\
\hline$s t d$ & & 89.9 & 22.3 & 146.7 & 895.6 & 2053.7 & 3394.7 \\
\hline 20 & & & & 146.7 & 880.7 & 1990.4 & 3264.5 \\
\hline 25 & & & & 141.7 & 768.3 & & \\
\hline 30 & & & & 98.3 & 238.1 & & \\
\hline 40 & & & & 18.2 & 40.2 & 27.6 & \\
\hline
\end{tabular}

$[\mathrm{Ba} / \mathrm{Fe}] \quad 0.5-2.0$

\begin{tabular}{llllll}
0. & 0. & $10^{-5}$ & 1.37 & 1.83 & 2.05 \\
\hline
\end{tabular}

${ }^{a}$ Estimated values for D461.

${ }^{b}$ Interpulse numbers corresponding to the labels reported in Fig. 2.

${ }^{c}$ Mass of dredged up material (in $M_{\odot}$ ).

${ }^{d}$ std refers to models without CBP (see text); 20, 25, 30 and 40 indicate $T_{\mathrm{CBP}}$, the maximum temperature of the $\mathrm{CBP}$, in $10^{6} \mathrm{~K}$.

with the products of the He burning ${ }^{3}$. The amount of mass dredged up is reported in Table 1. After a dredge up episode, the expanded layers fall back, the $\mathrm{H}$ burning restarts and the convective envelope recedes. Close to the zone of maximum penetration, the receding envelope leaves a variable profile of $\mathrm{H}$. At the $\mathrm{H}$ re-ignition, $\mathrm{a}^{13} \mathrm{C}$ pocket forms in this zone. The total mass of ${ }^{13} \mathrm{C}$ within the pocket ranges between $10^{-5} M_{\odot}$ (after the first TDU episode) and $10^{-6} M_{\odot}$ (last computed TDU). Then, during the interpulse, the temperature rises up, until the ${ }^{13} \mathrm{C}(\alpha, n){ }^{16} \mathrm{O}$ reaction takes place. This neutron source activates the s-process nucleosynthesis and then, heavy isotopes (up to Bi) are synthesized (Straniero et al. 1995; Gallino et al. 1998; Cristallo et al. 2004).

The resulting modification of the envelope composition is reported in Table 1.

The evolutionary track in the $\log g-\log T_{\text {eff }}$ diagram is shown in Fig. 3. The position of D461, as derived by best fitting the final spectrum, is also reported. The effective temperature of the models drops after the onset of the third dredge up. This

\footnotetext{
3 The thermal pulse generates a convective zone that extends from the He burning shell up to the top of the $\mathrm{H}$-exhausted region. Thus, $\mathrm{C}$ and s-process elements are efficiently mixed within this convective zone.
} 


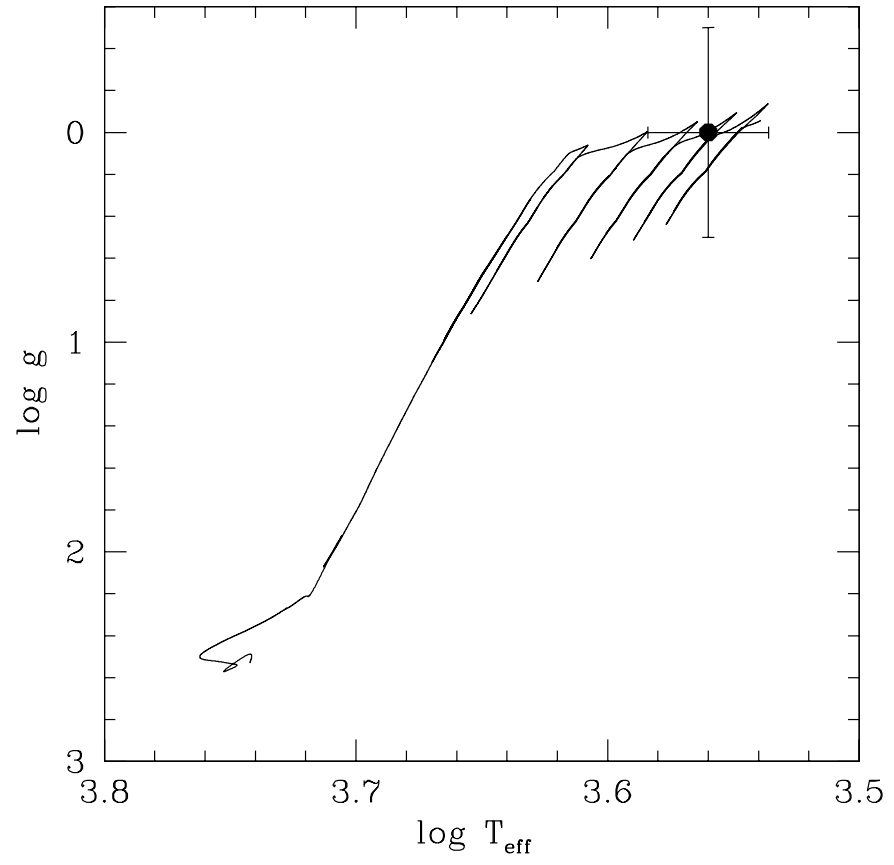

Fig. 3. Evolutionary track of $1.5 M_{\odot}(Z=0.0003)$ stellar model in the $\log g-\log T_{\text {eff }}$ diagram. The track starts at the beginning of the core He burning. Few thermal pulses are included. The position of D461 is also shown. It is consistent with a thermally pulsing low mass AGB star undergoing the third dredge up.

is due to the increase of the envelope opacity caused by the carbon enhancement (Marigo et al. 2002) ${ }^{4}$. The figure shows that the effective temperature of the AGB model approaches that estimated for D461 after a few dredge up episodes. The estimated gravity is also compatible with that of low mass TP-AGB stellar models. Note that due to the scarcity of $\mathrm{O}$ in the envelope, the $\mathrm{C} / \mathrm{O}$ ratio immediately grows above the unity (see Table 1 ). After two dredge up episodes the $\mathrm{C} / \mathrm{O}$ is about 10 . Here we have assumed scaled solar initial composition. An oxygen enhancement of a factor 2 or 3, or a larger metallicity of the initial model, would lead to a better agreement with the estimate of the $\mathrm{C} / \mathrm{O}$ ratio for $\mathrm{D} 461$. The lower limit for the carbon isotopic ratio and the constraint for the $[\mathrm{Ba} / \mathrm{Fe}]$ (see the previous section) are also fulfilled. On the contrary the lithium production cannot be explained with our standard model. This is because the temperature at the base of the convective envelope never attains $2 \times 10^{7} \mathrm{~K}$, the minimum temperature for the synthesis of Be.

This is a well known problem: some peculiar low mass AGB stars show low ${ }^{12} \mathrm{C} /{ }^{13} \mathrm{C}$ and high Li abundances, which are clear signatures of nuclear processes occurring at the base of the convective envelope (Abia \& Isern 1997). To solve such kind of problems, Wasserburg et al. (1995; see also Nollet et al. 2003) suggested that some amount of material could be transported from the fully convective envelope into the underlying

${ }^{4}$ We have simulated the change of the opacity caused by the dredge up by means of opacity tables with variable metallicity, but scaled solar mixture. This probably underestimates the contribution of some molecular species that form in a $\mathrm{C}$ rich mixture. Unfortunately, the available low temperature opacity tables does not include such effects. radiative region, down to the outer zone of the $\mathrm{H}$ burning shell. Although the actual physical basis for such phenomenon (that they called cool bottom process, $\mathrm{CBP}$ ) is not known, it has been invoked to explain some chemical anomalies of RGB stars and in studies of dust grains that formed in circumstellar envelopes (e.g., Huss et al. 1994; Boothroyd et al. 1994; Harris et al. 1985; Kahane et al. 1992).

Then we have computed some additional AGB stellar models by including a parameterized description of the deep circulation during the interpluse period. Following the scheme proposed by Nollet et al. (2003), we assume that the CBP depends on two free parameters, namely the maximum temperature reached by the circulating material $\left(T_{\mathrm{CBP}}\right)$ and the circulation velocity $\left(v_{\mathrm{CBP}}\right)^{5}$.

The effect on the surface composition is illustrated in Table 1. Models including the CBP are identified by the corresponding $T_{\mathrm{CBP}}$. The standard (std) model refers to the case without CBP. As a consequence of the cool bottom process, the abundance of Li initially increases, reaches a maximum and then, once the ${ }^{3} \mathrm{He}$ (the fuel for the Be production) is consumed, decreases. The better agreement with the measured $\mathrm{Li}$ abundance in D461 is obtained for a bottom temperature of 25-30 $\left(10^{6} \mathrm{~K}\right)$. Another consequence of the CBP is the partial consumption of ${ }^{12} \mathrm{C}$ and the enhancement of the ${ }^{13} \mathrm{C}$ in the envelope. This affects the carbon isotopic ratio and the $\mathrm{C} / \mathrm{O}$, which remain, in any case, within the derived observational constraints for D461.

\section{Conclusions}

Our analysis has been limited by the low spectral resolution and by the lack of reliable model atmospheres appropriate for the peculiar chemical composition of $\mathrm{D} 461$. The $\mathrm{C} / \mathrm{O}$, for example, is significantly larger than that measured in any other C-star. In spite of that, several features in the spectra of D461 converge toward the same conclusion: it is a thermally pulsing low mass $\left(\sim 1.5 M_{\odot}\right)$ low metallicity $([\mathrm{Fe} / \mathrm{H}] \sim-2)$ AGB star, undergoing the third dredge up. Gravity and effective temperature are also consistent with this hypothesis. This is the first

\footnotetext{
${ }^{5}$ Nollet et al. actually use the mass flux instead of the circulation velocity, but these two quantities are clearly related. Note that in Nollet et al. the effect of the CBP on the nucleosynthesis was evaluated by means of a post-process, whereas we have included this calculation in the stellar evolution code. In particular, we mimic the CBP with a deep-mixing obtained by means of the same algorithm used to describe the mixing in the convective zones (see Chieffi et al. 2001 for details). Thus, the extension and the efficiency of the deep-mixing are controlled by $T_{\mathrm{CBP}}$ and $v_{\mathrm{CBP}}$, respectively. In agreement with the previous investigation, we find that the key parameter is the maximum temperature reached by the circulating material. $T_{\mathrm{CBP}}$ has been varied from 20 to $40\left(10^{6} \mathrm{~K}\right)$. Within this range the maximum penetration in mass is of the order of $5 \times 10^{-3} M_{\odot}$ and the physical structure of the star is very marginally affected. In the zone where the CBP takes place, we have adopted a constant circulation velocity equal to a fraction (1, $1 / 10,1 / 100)$ of the mixing velocity in the most internal layers of the convective envelope. Typically the maximum explored values for $v_{\mathrm{CBP}}$ are of the order of $4 \times 10^{4} \mathrm{~cm} / \mathrm{s}$, which correspond to a mass flux of $0.05 M_{\odot} / \mathrm{yr}$. The minimum value is about 100 times smaller. Within this range, the resulting surface $\mathrm{Li}$ abundance is practically insensitive to a change of $v_{\mathrm{CBP}}$.
} 
discovery of an intrinsic $\mathrm{C}(\mathrm{N})$ star in a metal poor stellar population. The Li enhancement is likely due to a moderate cool bottom process.

This scenario implies that the mass of D461 cannot be smaller than $1.2 M_{\odot}$, because a less massive star would attain the thermally pulsing AGB phase with a too small envelope for the occurrence of the third dredge up. On the other hand, the observed visual magnitude (nearly that of the RGB tip) excludes the possibility that D461 could be a massive AGB star. We estimate that $2 M_{\odot}$ is a conservative upper limit for the mass of D461. Such a stringent limitation of the stellar mass implies a striking constraint to the age of D461: it should range between 1 and 3 Gyr (see e.g., Table 1 in Domínguez et al. 1999).

We remind, again, that the bulk of the stellar population of the Draco dwarf spheroidal is dominated by very old stars $(\sim 10 \mathrm{Gyr})$ although the star formation extended up to $2 \mathrm{Gyr}$ ago. This latter corresponds to the age of a $1.5 M_{\odot}$ AGB star (for the typical metallicities of Draco). Note that the anomalous Cepheids, which are currently detected in Draco, should have masses very close to this value (Bono et al. 1997).

Let us finally mention an alternative scenario. It could be possible that D461 was, in origin, a low mass $\operatorname{star}\left(M<1 M_{\odot}\right)$ whose mass has been recently accreted, as a consequence of a mass exchange or coalescence, from a companion in a close binary system. Up to about $200 \mathrm{Myr}$ ago D461 was an old main sequence star becoming a Blue Straggler and, more recently, a pulsating horizontal branch star with the characteristics of the anomalous Cepheids. Radial velocity monitoring of D461, by Olszewski et al. (1996), found no evidence of the existence of a compact companion, but Shetrone et al. (2001a) detected a possible photometric variability (at $4 \sigma$ level). Such a variability is, however, quite common among AGB stars belonging to intermediate-age populations. Additionally, note that the binary hypothesis is disfavoured because of the high $\mathrm{Li}$ abundance (see Abia et al. 1993). In summary, the presently available data cannot allow a definitive discrimination between the old and the young hypothesis for D461, even if, from a statistical point of view, the latter is the more significative.

Acknowledgements. Data from the VALD database at Vienna were used for the preparation of this paper. The $4.2 \mathrm{~m}$ WHT is operated on the island of La Palma by the RGO in the Spanish Observatory of the Roque de los Muchachos of the Instituto de Astrofísica de Canarias. This work has been partially supported by the MCyT grant AYA2002-04094-C03-03 and by the spanish-italian cooperation INFN-CICYT.

\section{References}

Aaronson, M., \& Mould, J. 1985, ApJ, 290, 191

Abia, C., \& Isern, J. 1996, ApJ, 460, 443

Abia, C., \& Isern, J. 1997, MNRAS, 289, L11

Abia, C., \& Isern, J. 2000, ApJ, 536, 438

Abia, C., Boffin, H. M. J., Isern, J., \& Rebolo, R. 1993, A\&A, 272, 455

Abia, C., Pavlenko, Ya. V., \& de Laverny, P. 1999, A\&A, 351, 273

Abia, C., Domínguez, I., Gallino, R., et al. 2002, ApJ, 579, 817

Aparicio, A., Carrera, R., \& Martínez-Delgado, D. 2001, ApJ, 122, 2524
Armandroff, T. E., Olszewski, E. W., \& Pryor, C. 1995, AJ, 110, 2131

Azzopardi, M., Muratorio, G., Breysacher, J., \& Westerlund, B. E. 1999, in Stellar populations in the Local Group, ed. P. Whithelock, \& R. Cannon (ASP), IAU Symp., 192, 144

Baade, W., \& Swope, H. H. 1961, AJ, 66, 300

Bell, R. A. 1985, PASP, 97, 219

Bellazzini, M., Ferraro, F. R., Origlia, L., et al. 2002, AJ, 124, 3222

Bono, G., Caputo, F., Santolamazza, P., Cassisi, S., \& Piersimoni, A. 1997, AJ, 113, 2209

Boothroyd, A., Sackmann, I. J., \& Wasserburg, G. J. 1994, ApJ, 430, 77

Cameron, A. G. W., \& Fowler, W. A. 1971, ApJ, 164, 111

Catchpole, R. M., \& Feast, M. W. 1976, MNRAS, 175, 501

Carney, B. W., \& Seitzer, P. 1986, AJ, 92, 23

Chieffi, A., Limongi, M., \& Straniero, O. 1998, ApJ, 502, 737

Chieffi, A., Domínguez, I., Limongi, M., \& Straniero, O. 2001, ApJ, 554,1159

Cristallo, S., Gallino, R., \& Straniero, O. 2004, Mem. Soc. Astron. Ital., 75, 124

Demers, S., Dallaire, M., \& Battinelli, P. 2002, AJ, 123, 3428

Domínguez, I., Chieffi, A., Limongi, M., \& Straniero, O. 1999, ApJ, 524, 226

Egan, M. P., van Dyk, S., \& Price, S. D. 2001, AJ, 122, 1844

Forestini, M., \& Charbonnel, C. 1997, A\&AS, 123, 241

Gallart, C., Freedman, W. L., Aparicio, A., Bertelli, G., \& Chiosi, C. 1999, AJ, 118, 2245

Gallino, R., Arlandini, C., Busso, M., et al. 1998, ApJ, 497, 388

García-Lario, P., D’Antona, F., Lub, J., Plez, B., \& Habing, H. J. 1999, in AGB Stars, ed. T. Le Bertre, A. Lebre, \& C. Waelkens, IAU Symp., 191, 91

Grillmair, C. J., Mould, J. R., Holtzman, J. A., et al. 1998, AJ, 115, 144

Groenewegen, M. A. 1999, in Asymptotic Giant Branch Stars, ed. T. Le Bertre, A. Lebre, \& C. Waelkens, IAU Symp., 191, 535

Harris, M. J., Lambert, D. L., \& Smith, V. V. 1985, ApJ, 292, 620

Harris, G. J., Pavlenko, Ya. V., Jones, H. R. A., \& Tennyson, J. 2003, MNRAS, 344, 1107

Hatzidimitriou, D., Morgan, D. H., Cannon, R. D., \& Croke, B. F. W. 2003, MNRAS, 341, 1290

Huss, G. R., Fahey, A. J., Gallino, R., \& Wasserburg, G. J. 1994, ApJL, 430,81

Iben, I., Jr., \& Renzini, A. 1983, ARA\&A, 21, 271

Ikuta, C., \& Arimoto, A. 2002, A\&A, 391, 55

Jorissen, A. 1999, in Asymptotic Giant Branch Stars, ed. T. Le Bertre, A. Lebre, \& C. Waelkens, IAU Symp., 191, 437

Kahane, C., Cernicharo, J., Gomez-Gonzalez, J., \& Guelin, M. 1992, A\&A, 256, 235

Knapp, G. R., Pourbaix, D., \& Jorissen, A. 2001, A\&A, 371, 222

Kupka, F., Piskunov, N., Ryabchikova, T. A., Stempels, H. C., \& Weiss, W. W. 1999, A\&AS, 138, 119

Kurucz, R. L. 1993, CD-ROM 18

Lambert, D. L., Gustafsson, B., Eriksson, K., \& Hinkle, K. H. 1986, ApJS, 62, 373

Lattanzio, J., \& Forestini, M. 1999, in Asymptotic Giant Branch Stars, ed. T. Le Bertre, A. Lebre, \& C. Waelkens, IAU Symp., 191

Lorenz-Martins, S. 1996, A\&A, 314, 209 (Dordrecht: Kluwer), 31

Margon, B., Anderson, S. F., Harris, H. C., et al. 2002, AJ, 124, 1651

Marigo, P. 2002, A\&A, 387, 507

Mateo, M. 1998, ARA\&A, 36, 345

Matsuura, M., Zijlstra, A. A., van Loon, J. Th., et al. 2002, ApJ, 580, L133

Munari, U. 1991, A\&A, 251, 103

Naersisian, S. E., Shavrina, A. V., \& Yaremchuk, A. A. 1989, Astrofisika, 20, 249 
Nollett, K. M., Busso, M., \& Wasserburg, G. 2003, ApJ, 582, 1036

Ohnaka, K., \& Tsuji, T. 1996, A\&A, 310, 933

Olszewski, E. W., Pryor, C., \& Armandroff, T. E. 1996, AJ, 111, 750

Pavlenko, Ya. V. 2003, Astron. Rept., 47, 59

Reyners, M., van Winckel, H., Biémont, E., \& Quintet, P. 2002, A\&A, 395, L35

Renzini, A., \& Voli, M. 1981, A\&A, 94, 175

Sackmann, I. J., \& Boothroyd, A. I. 1992, ApJ, 392, L71

Shetrone, M. D., Bolte, M., \& Stetson, P. B. 1998, ApJ, 115, 1888

Shetrone, M. D., Côte, P., \& Stetson, P. B. 2001a, PASP, 113, 1122

Shetrone, M. D., Côte, P., \& Sargent, W. L. 2001b, ApJ, 548, 592

Sneden, C., Johnson, H. R., \& Krupp, B. M. 1976, ApJ, 204, 218

Straniero, O., Gallino, R., Busso, M., et al. 1995, ApJ, 440, L85
Straniero, O., Chieffi, A., \& Limongi, M. 1997a, ApJ, 490, 425

Straniero, O., Chieffi, A., Limongi, M., et al. 1997b, ApJ, 478, 332

Smith, V. V., Plez, B., Lambert, D. L., \& Lubowich, D. A. 1995, ApJ, 441, 735

Travaglio, C., Randich, S., Galli, D., et al. 2001, ApJ, 559, 909

Vanture, A. D. 1992, AJ, 103, 2035

Wallerstein, G., \& Knapp, G. R. 1998, ARA\&A, 36, 369

Wasserburg, G. J., Boothroyd, A. I., \& Sackmann, I.-J. 1995, ApJ, 440, L101

Whitelock, P. A., Menzies, J., Irwin, M., \& Feast, M. 1999, in Stellar populations in the Local Group, ed. P. Whithelock, \& R. Cannon (ASP), IAU Symp., 192, 136 